\title{
PREVALÊNCIA CLÍNICA-EPIDEMIOLÓGICA DOS PACIENTES CIRÚRGICOS DE VARIZES EM MEMBROS INFERIORES
}

\section{CLINICAL-EPIDEMIOLOGICAL PREVALENCE OF PATIENTS UNDERGOING VARICE SURGERY IN LOWER MEMBERS}

\section{PREVALENCIA CLÍNICO-EPIDEMIOLÓGICA DE PACIENTES QUIRÚRGICOS CON VARICESEN MIEMBROS INFERIORES}

Thabata Coaglio Lucas ${ }^{1}$, Frederico Pelli Seabra ${ }^{2}$, Laura Petronilha Santos ${ }^{3}$, Ezequiel de Souza Almeida ${ }^{4}$, Lucas Souza e Costa ${ }^{5}$, Ana Paula Rodrigues ${ }^{6}$.

\section{RESUMO}

Objetivo: investigar a prevalência clínica-epidemiológica dos pacientes submetidos à cirurgia de varizes dos membros inferiores. Método: estudo transversal. Aplicou-se um questionário de qualidade de vida semiestruturado, adaptado do Venous Insufficiency Epidemiological and Economic Study, previamente à cirurgia e, após 40 dias da cirurgia, utilizou-se Teste de Qui-quadrado de Pearson e teste exato de Fisher, com correção de Bonferroni. As variáveis bivariadas foram consideradas significativas, quando geraram valor de $p<0,05$. Resultados: em $75,8 \%$ das mulheres havia veias perfurantes, antes da cirurgia, e 66 \% delas permaneciam com veias varicosas, após a cirurgia. $80 \%$ dos homens não apresentavam limitações, no trabalho, e $61,7 \%$ das mulheres não mostravam limitações em casa e no trabalho, no pós-cirúrgico. Uma prevalência de $72 \%$ dos participantes, que tinham trombose venosa profunda, foi associada à hipertensão arterial e ao diabetes $(p<0,05)$. Conclusão: os resultados apontaram para a necessidade de uma vigilância epidemiológica sistemática e ativa dos pacientes, que permaneceram com veias varicosas, mesmo após terem sido submetidos à cirurgia de varizes, em algum dos membros inferiores. A alta prevalência desses pacientes levou a um aumento do risco potencial de desenvolver trombose venosa profunda e consequente aumento dos eventos adversos físicos e psicológicos.

Descritores: Trombose; Procedimentos Cirúrgicos Vasculares; Indicadores (estatística); Epidemiologia; Enfermagem baseada em Evidências; Insuficiência Venosa.

\begin{abstract}
Objective: To investigate the clinical and epidemiological prevalence of patients undergoing varicose vein surgery. Method: cross-sectional study. A semi-structured quality of life questionnaire adapted from the Venous Insufficiency Epidemiological and Economic Study was applied prior to surgery and after 40 days of surgery. Pearson's chi-square test and Fisher's exact test with Bonferroni correction were used. Bivariate variables were considered significant when $p<0.05$. Results: a total of $75.8 \%$ of women had perforating veins before surgery and $66 \%$ of them had varicose veins after surgery. $80 \%$ of men had no limitations at work and $61.7 \%$ of women had no limitations at home and at work postoperatively. A prevalence of $72 \%$ of participants with deep vein thrombosis was associated with hypertension and diabetes $(p<0.05)$. Conclusion: The results pointed out the need for systematic and active epidemiological surveillance of patients who remained with varicose veins, even after undergoing varicose vein surgery on any of the lower body members. The high prevalence of these patients led to an increased potential risk of developing deep vein thrombosis and a consequent increase in physical and psychological adverse events.
\end{abstract}

Keywords: Thrombosis, Vascular Surgical Procedures; Indicators (Statistics); Epidemiology; Evidence-based Nursing; Venous Insufficiency.

\section{RESUMEN}

Objetivo: investigar la prevalencia clínica y epidemiológica de pacientes sometidos a cirugía de venas varicosas. Método: estudio transversal. Se aplicó un cuestionario semiestructurado de calidad de vida, adaptado del Estudio Epidemiológico y Económico de Insuficiencia Venosa, antes de la cirugía y después de 40 días de la cirugía. Se utilizaron la prueba de chi-cuadrado de Pearson y la prueba exacta de Fisher con corrección de Bonferroni. Las variables bivariadas se consideraron significativas cuando $p<0.05$. Resultados: $75,8 \%$ de las mujeres tenían venas perforantes antes de la cirugía y $66 \%$ de ellas tenían venas varicosas después de la cirugía. El $80 \%$ de los hombres no tenían limitaciones en el trabajo y el 61,7\% de las mujeres no tenían limitaciones en el hogar ni en el trabajo después de la operación. Una prevalencia del $72 \%$ de los participantes con trombosis venosa profunda se asoció con hipertensión y diabetes ( $p<0,05$ ). Conclusión: los resultados señalaron la necesidad de una vigilancia epidemiológica sistemática y activa de los pacientes que permanecieron con venas varicosas, incluso después de someterse a una cirugía de venas varicosas en cualquiera de las extremidades inferiores. La alta prevalencia de estos pacientes condujo a un mayor riesgo potencial de desarrollar trombosis venosa profunda y al consiguiente aumento de eventos adversos físicos y psicológicos.

Descriptores: Trombosis; Procedimientos Quirúrgicos Vasculares; Indicadores (Estadística); Epidemiología; Enfermería Basada en la Evidencia; Insuficiencia Venosa.

'Doutora em Engenharia Mecânica/Bioengenharia pela Universidade Federal de Minas Gerais. Professora do Departamento de Enfermagem da Universidade dos Vales do Jequitinhonha e Mucuri. Diamantina - UFVJM, MG, Brasil. ${ }^{2}$ Médico. Cirurgião vascular. Professor do Departamento de Medicina da UFVJM. ${ }^{3}$ Enfermeira pela UFVJM. ${ }^{4}$ Discente do Curso de Medicina da UFVJM. ${ }^{5}$ Discente do Curso de Medicina da UFVJM. ${ }^{6}$ Doutora em Farmácia pela Universidade Estadual Paulista Júlio de Mesquita Filho. Professora Associada do Departamento de Farmácia da UFVJM. 


\section{INTRODUÇÃO}

A Insuficiência Venosa Crônica (IVC) impacta a saúde pública, em todo o mundo, e gera custos financeiros para os serviços de saúde, aumenta a morbi-mortalidade, reduz a qualidade de vida e aumenta o absenteísmo ${ }^{(1)}$. Nos Estados Unidos, cerca de sete milhões de norte americanos são acometidos pela IVC, o que corresponde de 70 a $90 \%$ de todas as úlceras de membros inferiores, na população ${ }^{(1)}$.

No Brasil, a IVC ocupa a 14a posição, no que se refere ao afastamento laboral, podendo levar, até mesmo, à aposentadoria precoce ${ }^{(2)}$. Dados epidemiológicos apontam a prevalência de IVC em $35,5 \%$ da população, sendo que $1,5 \%$ é afetada pela úlcera venosa ${ }^{(3)}$.

A IVC é conhecida como um conjunto de fenômenos clínicos, decorrentes de um desequilíbrio funcional do sistema venoso, determinado por hipertensão venosa, geração de refluxo e obstrução do fluxo venoso ${ }^{(3)}$. Esse desequilíbrio pode ser congênito ou adquirido, podendo atingir os sistemas venosos superficiais e profundos. A hipertensão do sistema venoso é a responsável pelo surgimento dos sintomas clínicos dessa enfermidade ${ }^{(4)}$.

Estudos apontam que a IVC pode causar tanto prejuízos funcionais no individuo quanto também afetar a sua qualidade de vida, decorrente de seus sinais vitais e sintomas, tais como: dor, edema, lipodermatoesclerose e dificuldade para caminhar e realizar atividades diárias de vida ${ }^{(3-5)}$.

A qualidade de vida está relacionada à sua independência funcional, que se refere à sua autonomia e à sua capacidade de se relacionar com o meio ${ }^{(3)}$. A IVC e seus fatores associados, como a úlcera venosa, possuem como fator de risco em comum o aumento da idade, o que pode levar à diminuição da capacidade funcional e produtiva do indivíduo ${ }^{(6)}$.

Em razão da ineficácia das ações de redução de morbi-mortalidade e ausência da repercussão dos benefícios dos cuidados de saúde, na manifestação da Doença Venosa Crônica (DVC), a busca pelo conhecimento científico e clínico da doença, e de seus fatores associados, tem aumentado de forma significativa no Brasil $^{(7)}$. Tais ações teriam o intuito de redução dos gastos, provenientes do tratamento dessa patologia, e diminuição da incidência das suas formas graves, por meio de uma avaliação precoce e eficiente.
Outro aspecto relevante é que, embora seja uma realidade mundial, o aumento da prevalência e da gravidade da DVC, estudos a respeito da intensidade dos sintomas e do comprometimento à qualidade de vida dos indivíduos, acometidos pela DVC, ainda são escassos ${ }^{(3,5,7)}$. Ademais, apesar de estudos apontarem que $\mathrm{o}$ uso de um questionário validado como VEINES QoL/Sym possibilita um maior conhecimento da qualidade de vida do individuo com IVC e de seu impacto funcional, psicológico e social( ${ }^{(4,6)}$, poucos estudos confrontam a gravidade dos sinais clínicos e o desenvolvimento da doença do indivíduo, atingido por essa patologia ${ }^{(4,6)}$.

Há, no entanto, uma lacuna no conhecimento científico, na descrição do perfil epidemiológico dos pacientes pré e póscirúrgico, bem como o impacto do tratamento em sua qualidade de vida. Dessa forma, neste estudo, objetivou-se avaliar o perfil clínicoepidemiológico dos pacientes, com abordagem cirúrgica de membros inferiores.

\section{MÉTODO}

Estudo transversal, realizado num hospital filantrópico, no interior de Minas Gerais. A seleção dos participantes foi feita por conveniência, composta por pacientes diagnosticados com IVC e com indicação de tratamento cirúrgico, internados, no período de agosto de 2017 a novembro de 2018.

No período de estudo, estiveram internados 260 pacientes com IVC. No entanto, foram incluídos, neste estudo, 62 participantes que fizeram cirurgia vascular.

O cálculo do tamanho da amostra foi realizado, considerando o desenho metodológico do tipo transversal, usando o software Epidat versão 4.1 (Organização Pan-Americana de Saúde Pública), fixando os seguintes parâmetros: proporção esperada de $50 \%$, em razão da heterogeneidade das variáveis a serem mensuradas; a margem de erro tolerável foi de $5 \%$ e nível de confiança, de $95 \%$. O tamanho amostral foi ajustado para populações finitas e, ao número definido, foram acrescidos $30 \%$, para compensar possíveis perdas ou recusas, e $20 \%$ para aumentar o poder estatístico dos testes de comparação de proporções.

Os critérios de inclusão para este estudo foram: participantes diagnosticados com IVC e com indicação de tratamento cirúrgico (Classification System for Chronic Venous 
Disorders (CEAP) 2 à 6); faixa etária entre 25 a 85 anos e de ambos os sexos.

Os critérios de exclusão foram: participantes que foram avaliados pelo cardiologista ou anestesiologista, na avaliação pré-anestésica, como pertencentes à American Society of Anesthesiologists (ASA III ou acima), isto é, aqueles que apresentavam doenças sistêmicas graves, incapacitantes ou não, com ameaça grave à vida, e participantes diagnosticados com problemas cognitivos.

Aplicou-se um questionário semiestruturado, adaptado no Venous Insufficiency Epidemiological and Economic (VEINES QoL /Sym $\left.)^{\circ}\right)^{(3-5)}$, em dois momentos: précirúrgico e, 40 dias, pós-cirúrgico.

Para este estudo consideraram-se as seguintes variáveis: Pré-cirúrgicas: a) Sociodemográficas: sexo, idade, presença de TVP anterior, HAS, diabetes, histórico de cirurgia anterior, tabagista, etilista, exercicios físicos b) Classificação clínica: 1-CEAP: CO: Sem sinais visíveis ou palpáveis da doença; C1: Telangiectasias e/ou veias reticulares; C2: Veias varicosas; C3: Veias varicosas mais edema; C4: hiperpigmentação, edema, lipodermatoesclerose ou atrofia branca; C5: úlcera venosa cicatrizada e C6: úlcera ativa. 2- Etiologia: congênita, primária, secundária ou sem causa definida. 3-Anatomia: veias superficiais, profundas ou perfurantes. 4Fisiopatologia: refluxo, obstrução, refluxo e obstrução. c) Classificação física: dor, presença de veias varicosas, edema, hiperpigmentação, úlcera aberta e terapia compressiva. d) Limitações no trabalho em casa; e) Fatores psicológicos presentes no VEINES QoL $/$ Sym $^{(3-5),}$ como a preocupação com a aparência, preocupados em tombar nos objetos e, assim, sentem-se um peso para a família.

Para a aplicação dos questionários, estudantes de graduação, em medicina e enfermagem, da Universidade Federal dos Vales do Jequitinhonha e Mucuri (UFVJM), foram devidamente treinados por profissional especializado. $O$ treinamento foi estruturado nas seguintes etapas: leitura teórica e discussão de textos dos instrumentos utilizados e acompanhamento de exames clínicos e físicos com médico angiologista.

A aplicação do questionário deu-se numa sala reservada e foi garantido sigilo da identificação do participante. Os questionários foram codificados, permitindo a não identificação do participante. Aqueles que aceitaram participar da pesquisa assinaram o Termo de Consentimento Livre e Esclarecido (TCLE).

As análises foram realizadas com o auxílio do programa estatístico IBM SPSS ${ }^{\bullet}$ Statistics versão 20. Para a comparação dos dados, foi realizado teste Qui-quadrado de Pearson e o teste exato de Fisher. Os resultados foram considerados significantes, quando o nível de significância ( $p$ ) foi menor que 0,05. Para as variáveis com mais de duas categorias e que foram significativas, utilizou-se $\mathrm{o}$ teste de correção de Bonferroni. O presente estudo foi aprovado pelo Comitê de Ética em Pesquisa da UFVJM, sob o número de parecer 2.282.654 e CAAE 7215741700005108.

\section{RESULTADOS E DISCUSSÃO}

No presente estudo, verificou-se que o CEAP foi significativo $(p<0,003)$, quando se comparou o período pré e pós-cirúrgico. A maior parte dos participantes da pesquisa eram C2, $87,2 \%$, no período pré-cirúrgico, sendo que a prevalência reduziu para $12,8 \%$, no pós-cirúrgico.

Os pacientes cirúrgicos que eram C6 $(4,8 \%)$ passaram para classificação clínica $C 5$, no póscirúrgico. Esta é uma realidade que pode ocorrer, quando pacientes com TVP postergam a realização de cirurgias e deixam agravar o quadro clínico das veias varicosas e, muitas vezes, o procedimento cirúrgico pode não eliminar o problema, sobretudo, quando já se encontra instalada uma úlcera aberta e ativa nos membros inferiores.

A fisiopatologia foi significativa $(p<0,08)$ com a diminuição da prevalência do refluxo de $60 \%(48 / 80)$ para $40 \%(32 / 80)$. A prevalência de refluxo e obstrução se manteve, mas, no geral, as veias varicosas, no pós-cirúrgico, não apresentavam mais refluxo.

Um estudo prospectivo avaliou pacientes femininos com IVC no pós-cirúrgico(1). Verificouse que, no pós-operatório, houve melhoria na classificação CEAP em 11 pacientes, dos 15 avaliados, sendo que um dos pacientes apresentou melhora da classificação CEAP, em apenas um dos membros $(p<0,001)^{(1)}$. Neste estudo, por outro lado, houve uma limitação nesse sentido, pois, apesar da melhora do CEAP, após a cirurgia, os participantes foram classificados, clinicamente, somente na perna em que a cirurgia foi realizada. Não se avaliou o CEAP da perna em que não foi feita a cirurgia, sendo assim, o sucesso ou recidiva da cirurgia foi 
considerado na mesma perna em que já havia sido feito o procedimento cirúrgico.

Na Tabela 1, apresenta-se a distribuição das variáveis associadas às características clínicas (CEAP, localização anatômica, fisiopatologia) e físicas (dor, veias varicosas, edema, hiperpigmentação, lipodermatoesclerose, úlceras abertas, terapia de compressão), versus o período pré e pós-cirúrgico.

Tabela 1 - Análise de associação das características clínicas (CEA P, localização anatômica, fisiopatologia) e físicas (dor, veias varicosas, edema, hiperpigmentação, lipodermatoesclerose, úlceras abertas e terapia de compressão) versus o período pré e pós-cirúrgico. Diamantina/Mg/Brasil,2018.

\begin{tabular}{|c|c|c|c|}
\hline \multirow[t]{3}{*}{ Variáveis } & \multicolumn{2}{|r|}{ Cirurgia } & \multirow[t]{3}{*}{ P-valor } \\
\hline & Pré $(n=62)$ & Pós (n=62) & \\
\hline & $f(\%)$ & $f(\%)$ & \\
\hline \multicolumn{4}{|l|}{ CEAP * } \\
\hline $\mathrm{CO} * *(1)$ & ---- & $12(100)$ & ---- \\
\hline $\mathrm{C} 19$ & --- & $41(100)$ & 0,065 \\
\hline $\mathrm{C}^{\ddagger}$ & $41(87,2)$ & $6(12,8)$ & 0,034 \\
\hline $\mathrm{C3}^{+}$ & $8(12,9)$ & --- & 0,014 \\
\hline $\mathrm{C} 4^{\dagger}$ & $4(6,5)$ & ---- & $0,001 * * *$ \\
\hline C5 & $6(66,7)$ & $3(33,3)$ & $0,002 * * *$ \\
\hline$C 6^{\ddagger \ddagger}$ & ---- & $3(4,8)$ & 0,233 \\
\hline Localização anatômica & $0,003 \|$ & & \\
\hline Superficiais & $47(46,5)$ & $54(53,5)$ & \\
\hline Perfurantes & $5(62,5)$ & $3(37,5)$ & \\
\hline \multicolumn{4}{|l|}{ Fisiopatologia } \\
\hline Refluxo & $48(60,0)$ & $32(40,0)$ & $0,005\|\|$ \\
\hline Refluxo e obstrução(1) & $2(50,0)$ & $2(50,0)$ & ---- \\
\hline Ausência de Refluxo & $12(30,0)$ & $28(70,0)$ & $0,04\|\|$ \\
\hline \multicolumn{4}{|l|}{ Dor } \\
\hline Nenhuma(1) & ---- & $30(100,0)$ & ---- \\
\hline Muito leve & ---- & $18(100,0)$ & 0,010 \\
\hline Leve & $5(38,4)$ & $9(61,6)$ & 0,023 \\
\hline Moderada & $23(82,1)$ & $5(17,9)$ & $0,003^{\S}$ \\
\hline Grave & $20(100,0)$ & ---- & 0,054 \\
\hline Muito grave & $14(100,0)$ & ---- & 0,046 \\
\hline Veias varicosas & & & 0,960 \\
\hline Sim & $62(62,0)$ & $38(38,0)$ & \\
\hline Não & ---- & $24(100,0)$ & \\
\hline Edema & & & 0,350 \\
\hline Sim & $35(71,4)$ & $14(28,6)$ & \\
\hline Não & $27(36,0)$ & $48(64,0)$ & \\
\hline Hiperpigmentação & & & 0,430 \\
\hline Sim & $36(69,3)$ & $16(30,7)$ & \\
\hline Não & $26(36,0)$ & $46(64,0)$ & \\
\hline Lipodermatoesclerose & & & 0,640 \\
\hline Sim & $9(90,0)$ & $1(10,0)$ & \\
\hline Não & $53(46,4)$ & $61(53,6)$ & \\
\hline Úlceras abertas & & & $0,030 \|$ \\
\hline Sim & $3(50,0)$ & $3(50,0)$ & \\
\hline Não & $59(50,0)$ & $59(50,0)$ & \\
\hline Terapia de compressão & & & 0,560 \\
\hline Sim & $8(13,6)$ & $51(86,4)$ & \\
\hline Não & $54(83,0)$ & $11(17,0)$ & \\
\hline
\end{tabular}

Fonte: Elaborada pelos próprios autores.

*Classification System for Chronic Venous Disorders; **Sem sinais visíveis ou palpáveis da doença; Telangiectasias e/ou veias reticulares; ${ }^{\ddagger}$ Veias varicosas; ${ }^{\dagger}$ Veias varicosas mais edema; ${ }^{\dagger}$ hiperpigmentação,edema, lipodermatoesclerose ou atrofia branca; 「úlcera venosa cicatrizada; $¥ u$ ulcera ativa;**Nível de significância $(\mathrm{P}<0,003)$-Teste Qui-quadrado de Pearson com correção de Bonferroni, sendo o número(1) a variável de referência para a análise."Nível de significância considerando Teste qui-quadrado $(p<0,05)$. IIIN Nível de significância $(P<0,08)$-Teste Qui-quadrado de Pearson com correção de bonferroni. §Nível de significância $(\mathrm{P}<0,004)$-Teste Qui-quadrado de Pearson com correção de Bonferroni.

Verificou-se, neste estudo, que do total de participantes $(n=62), 55(87,7 \%)$ tinham etiologia primária, antes da cirurgia, e $46(74,2 \%), 40$ dias, pós-cirurgia. $7(11,3 \%)$ apresentavam etiologia secundária, antes da cirurgia e $5(8,1 \%)$, no póscirúrgico $(p=0,003)$. 
Os resultados do presente estudo apontaram uma associação, estatísticamente, significativa entre apresentar trombose venosa profunda e ser hipertenso e diabético $(P<0,05)$.
Na Tabela 2, apresenta-se a associação entre as variáveis, hipertensão arterial, diabetes, tabagista e etilista, versus tromboflebite.

Tabela 2 - Distribuição da associação entre tromboflebite versus hipertensão arterial sistêmica, diabetes, tabagista e etilista. Diamantina/Mg/Brasil,2018.

\begin{tabular}{|c|c|c|c|}
\hline \multirow[b]{2}{*}{ Variáveis } & \multicolumn{2}{|c|}{ Trombose Venosa Profunda } & \multirow[b]{2}{*}{ P-valor } \\
\hline & $\begin{array}{c}\text { Sim } \\
(n=37) \\
f(\%)\end{array}$ & $\begin{array}{c}\text { Não } \\
(n=25) \\
f(\%)\end{array}$ & \\
\hline \multicolumn{4}{|c|}{ Hipertensão Arterial sistêmica } \\
\hline Sim & $26(72)$ & $10(28)$ & \multirow{2}{*}{$0,03^{*}$} \\
\hline Não & $11(42,3)$ & $15(57,7)$ & \\
\hline \multicolumn{4}{|l|}{ Diabetes } \\
\hline Sim & $10(45,4)$ & $12(54,6)$ & \multirow{3}{*}{$0,04 *$} \\
\hline Não & $27(67,5)$ & $13(32,5)$ & \\
\hline \multicolumn{3}{|l|}{ Tabagista } & \\
\hline Sim & $5(83,3)$ & $1(16,7)$ & \multirow{3}{*}{$0,046 * *$} \\
\hline Não & $32(57)$ & $24(43)$ & \\
\hline \multicolumn{3}{|l|}{ Etilista } & \\
\hline Sim & $2(40)$ & $3(60)$ & \multirow{2}{*}{0,694} \\
\hline Não & $35(61,4)$ & $22(38,6)$ & \\
\hline
\end{tabular}

Fonte: Elaborada pelos próprios autores.

*Nível de significância $(p<0,05):$ *Teste Qui-quadrado de Pearson;**Teste Exato de Fisher.

Na Tabela 2, mostrou-se que $72 \%$ dos participantes, com TVP, também eram portadores de HAS e, $45,4 \%$ de diabetes $(p<0,05)$. Além disso, apesar do número pequeno de tabagistas, neste estudo, 83,3\% fumavam e apresentavam TVP. Em consonância com os resultados apontados acima, um estudo de meta análise também encontrou, em estudos prospectivos, que a hipertensão arterial sistêmica estava associada à TVP, com valor de risco relativo de 0,97 (IC=95\%:0,88-1,08) ${ }^{(10)}$. Além disso, a TVP também foi associada ao diabetes, com risco relativo de 1,01 (IC=95\%:0,89-1,15), e ao tabagismo, com risco relativo de 1,19 $(I C=95 \%: 1,08-1,32)^{(10)}$.

A TVP é a maior causa de óbitos intrahospitalares no mundo e, facilmente evitável, por meio da identificação de seus fatores de risco e de sua profilaxia ${ }^{(12)}$. Em um estudo, realizou-se ultrassonografia em pacientes suspeitos de TVP e identificaram-se alguns fatores de risco para desenvolver trombose como a idade de 41 a 60 anos, obesidade, história familiar de trombose e o uso de anticoncepcional(12) . Esses fatores de risco e a faixa etária também foram apontados como indicadores de saúde, no presente estudo, e associados ao desenvolvimento de TVP ${ }^{(12)}$.

Os resultados do presente estudo também corroboraram com outro estudo transversal em que, após a aplicação do questionário de Qualidade de Vida Relacionada à Saúde (QVRS), verificou-se que $61,4 \%$ dos participantes, com veias varicosas eram mulheres e com faixa etária maior que 60 anos $^{(5)}$.

A TVP pode desenvolver, em decorrência da incompetência valvular ou obstrução com interrupção do fluxo sanguíneo de retorno venoso, nas veias profundas dos membros inferiores, fator que gera hipertensão venosa e compromete a irrigação sanguínea dos tecidos no membro afetado, podendo levar ao surgimento de uma úlcera venosa ${ }^{(13)}$.

O histórico de cirurgia anterior associada à trombose venosa profunda, CEAP, presença de veias varicosas pré e pós-cirúrgica está apresentado na Tabela 3. 
Tabela 3 - Distribuição da etiologia, o CEAP, o histórico de cirurgia anterior e a presença de veias varicosas pré e pós-cirúrgico associados ao sexo em Diamantina/ MG/Brasil,2018.

\begin{tabular}{|c|c|c|c|}
\hline Variáveis & & Sexo & P-valor \\
\hline & $\begin{array}{c}\text { Feminino } \\
(n=47) \\
f(\%)\end{array}$ & $\begin{array}{c}\text { Masculino } \\
(n=47) \\
f(\%)\end{array}$ & \\
\hline $\begin{array}{l}\text { Etiologia pré- cirúrgica } \\
\text { Congênita }\end{array}$ & ---- & ---- & 0,093 \\
\hline Primária secundária & $41(74,5)$ & $14(25,5)$ & \\
\hline Sem causa & $6(85,7)$ & $1(14,3)$ & \\
\hline \multicolumn{4}{|l|}{ Etiologia pós-cirúrgica } \\
\hline Primária & $38(82,6)$ & $8(17,4)$ & $0,067^{*}$ \\
\hline Secundária(1) & $5(83,3)$ & $1(16,7)$ & ---- \\
\hline Sem causa & $4(40,0)$ & $6(60,0)$ & $0,045^{*}$ \\
\hline \multicolumn{4}{|l|}{ CEAP**pré-cirúrgico } \\
\hline $\mathrm{C} 2^{\ddagger}$ & $31(75,6)$ & $10(24,4)$ & \\
\hline $\mathrm{C3}^{+}$ & $4(57,1)$ & $3(42,9)$ & \\
\hline $\mathrm{C}^{+}$ & $5(100,0)$ & --- & 0,056 \\
\hline C5ף & $4(66,7)$ & $2(33,3)$ & \\
\hline$C 6^{\ddagger \ddagger}$ & $3(100,0)$ & ---- & \\
\hline \multicolumn{4}{|l|}{ CEAP**pós-cirúrgico } \\
\hline $\operatorname{co}^{\top}(1)$ & $5(41,7)$ & $7(58,3)$ & 0,003 ขึา \\
\hline $\mathrm{C} 1^{\prime}$ & $36(87,8)$ & $5(12,2)$ & $0,002^{\text {กाก }}$ \\
\hline $\mathrm{C}^{\ddagger}$ & $3(50,0)$ & $3(50,0)$ & $0,001^{\text {กาก }}$ \\
\hline C5ף & $3(100,0)$ & ---- & 0,310 \\
\hline \multicolumn{4}{|c|}{ Histórico de cirurgia anterior } \\
\hline Sim & $26(86,7)$ & $4(13,3)$ & $0,040\|\|$ \\
\hline Não & $21(65,6)$ & $11(34,4)$ & \\
\hline \multicolumn{4}{|c|}{ Veias varicosas pré-cirúrgicas } \\
\hline $\operatorname{Sim}$ & $47(75,8)$ & $15(24,2)$ & ----- \\
\hline Não & ---- & ---- & \\
\hline \multicolumn{4}{|c|}{ Veias varicosas pós-cirúrgicas } \\
\hline $\operatorname{Sim}$ & $31(81,6)$ & $7(18,4)$ & 0,165 \\
\hline Não & $16(66,7)$ & $8(33,3)$ & \\
\hline
\end{tabular}

Fonte: Elaborada pelos próprios autores.

* Nível de significância $(p<0,08)$ : Teste Exato de Fisher com correção de bonferroni, sendo o número(1) a variável de referência para a análise; ${ }^{* *}$ Classification System for Chronic Venous Disorders; ${ }^{\ddagger}$ Veias varicosas; ${ }^{\dagger}$ veias varicosas mais edema; ${ }^{\dagger}$ hiperpigmentação,edema, lipodermatoesclerose ou atrofia branca;" úlcera venosa cicatrizada; ${ }^{\ddagger \neq}$ úlcera ativa; 'Sem sinais visíveis ou palpáveis da doença;(Telangiectasias e/ou veias reticulares. "१ाTeste exato de Fisher com correção de Bonferroni $(p<0,03)$; IIITeste Qui-quadrado $(p<0,05)$.

Verificou-se que $75,8 \%$ das mulheres apresentavam veias varicosas, antes da cirurgia. No entanto, uma prevalência de $81,6 \%$ se mostravam com veias varicosas, após a cirurgia (Tabela 3). Cabe destacar que, apesar da melhora da classificação, ainda houve uma prevalência de C5 do sexo feminino, no pós-cirúrgico. A classificação clínica C5 pode possibilitar a ocorrência de recidiva da úlcera, uma vez que ainda existem veias varicosas, edema, hiperpigmentação, lipodermatoesclerose ${ }^{(5,12)}$. Por outro lado, do total de homens, $18,4 \%$ ainda permaneceram com veias varicosas, após a cirurgia.

As prevalências verificadas, na Tabela 3, podem justificar a presença de dor, edema, inflamação e hiperpigmentação, mesmo após a cirurgia de varizes. Esses sinais físicos são indicadores de saúde, que contribuem para uma readequação da saúde, aos cuidados diários com os membros inferiores, incluindo, descanso com 
pernas elevadas e atividades físicas regularmente.

A prevalência das mulheres, que apresentavam histórico de cirurgia anterior, foi de $86,7 \%$ (26/30) e dos homens foi de 3,3\%(4/30) $(p<0,05)$. Um estudo transversal aplicou um questionário de QVRS/SF-36 em pacientes com $D_{V}{ }^{(13)}$. Como nos resultados apresentados, neste estudo, a maioria era do sexo feminino, (69\%) quando comparado ao sexo masculino $(31 \%)^{(13)}$.A presença de DVC associada à úlcera venosa, em mulheres, é recorrente, já que as mulheres são mais propícias a determinados fatores de risco, tal como: o uso de contraceptivos, gravidezes múltiplas, problemas hormonais e baixa quantidade de massa muscular, que contribuem para uma maior tendência ao aparecimento de úlceras venosas ${ }^{(14)}$.

No estudo transversal, ao qual se refere $\operatorname{acima}^{(13)}$, a presença de úlcera venosa foi mais frequente entre os participantes com mais de 60 anos $(p=0,009)^{(13)}$. No presente estudo, também os idosos, femininos, com mais de 60 anos apresentaram as úlceras venosas abertas, com classificação em C6 ( $p=0,002)$, no pré-cirúrgico. Após 40 dias, no entanto, já estavam cicatrizadas e com classificação C5.

No presente estudo, verificou-se que, apesar da realização de cirurgias, os participantes apresentavam algum tipo de limitação nas atividades do trabalho e de casa. A idade foi significativa $(p<0,004)$, no que diz respeito à limitação no trabalho (Tabela 4). Com relação à idade, 30,3\% dos participantes, na faixa etária entre 40 e 59 anos, sentiam-se um pouco limitados, porém, nas atividades de casa, somente $18,2 \%$ tinham limitação, nessa mesma faixa etária.

Tabela 4 - Distribuição das limitações diárias após cirurgia, nas atividades de trabalho e de casa associadas ao sexo e a idade em Diamantina/MG/Brasil, 2018.

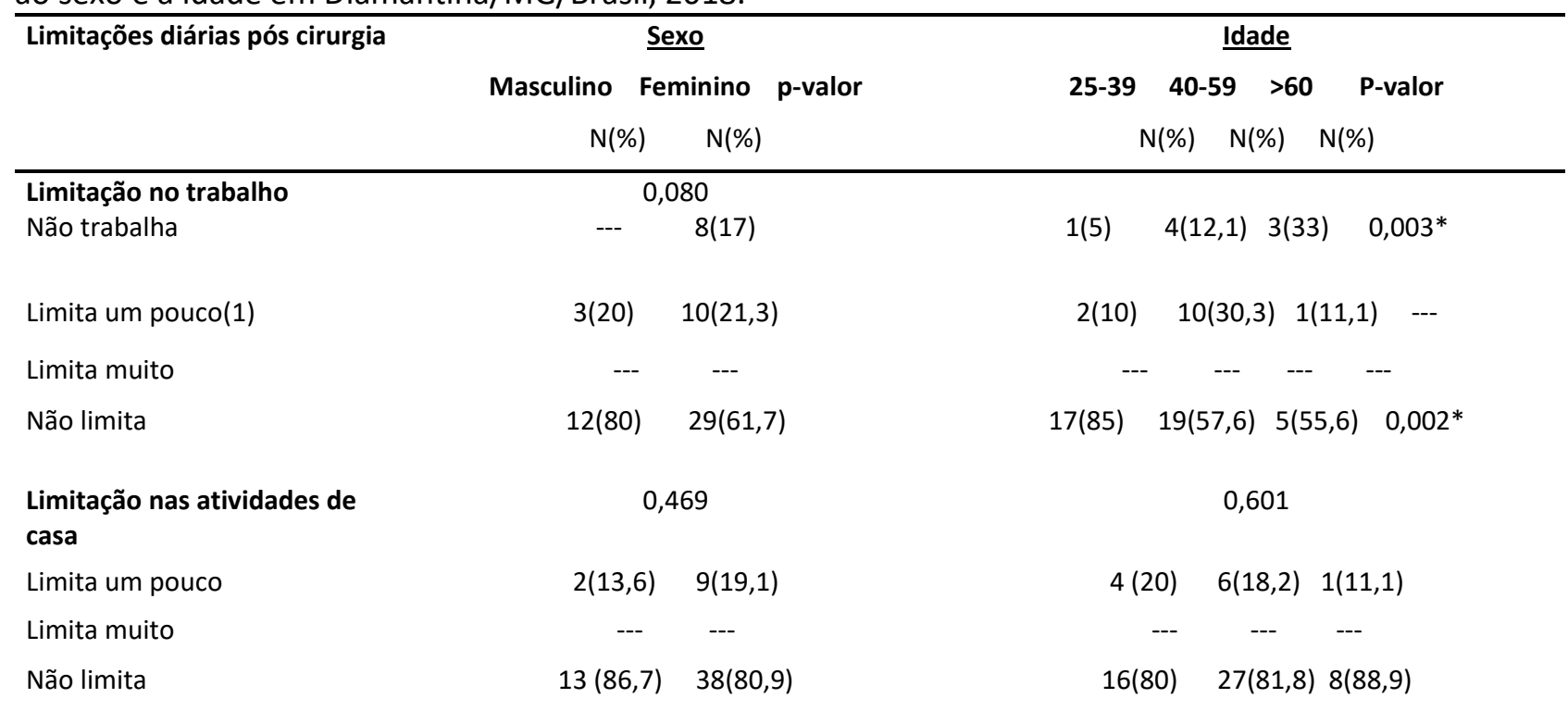

Fonte: Elaborada pelos próprios autores

*Nível de significância $(p<0,004)$ :Teste Exato de Fisher com correção de bonferroni com variável de referência identificada pelo número (1)

Em consonância com os resultados encontrados, um estudo transversal avaliou a qualidade de vida dos pacientes portadores de úlceras venosas, por meio de pontuações, em um instrumento de coleta de dados validados ${ }^{(15)}$. A limitação das atividades diárias de vida desses indivíduos criou uma sensação de desconforto, porque a maioria dos pacientes eram ativos e engajados em atividades, em seu trabalho ou em sua própria casa, antes de desenvolver a ferida (15).
Outro estudo realizado, nos Estados Unidos, que avaliou a qualidade de vida dos participantes portadores de veias varicosas e seu impacto nas suas atividades de vida diária reforça os achados encontrados, neste estudo ${ }^{(16)}$. O estudo também aplicou o questionário VEINESQOL/Sym ${ }^{\circledast}$ e verificou que, $47 \%$ dos participantes relataram dificuldade em realizar trabalho, $37 \%$ estavam limitados ao trabalho, $37 \%$ relataram ser limitado um pouco e $31 \%$ narraram reduzir o tempo no trabalho ${ }^{(16)}$. 
Quanto ao sexo, $80 \%$ dos homens não tinham limitações nas atividades do trabalho e $61,7 \%$ das mulheres não mostravam limitações. $17,7 \%$ dos participantes, que realizaram cirurgia, ainda apresentavam um pouco de limitação, nas atividades de trabalho. Tais grupos de participantes eram aqueles que ainda mantinham a presença de veias varicosas, após a cirurgia, seja pela idade avançada, seja pelo número de veias danificadas que, em razão da grande quantidade, não foi possível a remoção em uma única cirurgia.
Quanto aos aspectos psicológicos, apesar de não apresentarem significância $(p>0,05)$, no pós-cirúrgico, ainda havia participantes que se sentiam um peso para a família e que se preocupavam com a aparência das pernas e com a escolha da roupa. Além disso, os resultados envolvendo questões psicológicas, associadas ao sexo, apesar de não significativo $(P>0,05)$, demonstraram diferenças de prevalência que podem gerar indicadores de avaliação (Tabela 5).

Tabela 5 - Distribuição das questões psicológicas associadas ao sexo em Diamantina/MG/Brasil,2018.

\begin{tabular}{|c|c|c|c|}
\hline \multirow[t]{2}{*}{ Questões psicológicas } & \multicolumn{2}{|c|}{ Sexo } & \multirow[t]{2}{*}{ P-valor* } \\
\hline & Feminino & Masculino & \\
\hline Preocupado com a aparência das pernas & $N(\%)$ & $N(\%)$ & 0,153 \\
\hline O tempo todo & $3(6,38)$ & 0 & \\
\hline A maior parte do tempo & $2(4,25)$ & 0 & \\
\hline Boa parte do tempo & 0 & 0 & \\
\hline Alguma parte do tempo & $13(27,65)$ & 0 & \\
\hline Um pouco do tempo & $20(42,55)$ & $4(26,66)$ & \\
\hline Tempo nenhum & $9(19,78)$ & $11(73,34)$ & \\
\hline Total & 47(100) & $15(100)$ & \\
\hline A aparência influencia na escolha da roupa & & & 0,080 \\
\hline O tempo todo & $3(6,38)$ & 0 & \\
\hline A maior parte do tempo & $4(8,51)$ & 0 & \\
\hline Boa parte do tempo & $2(4,25)$ & 0 & \\
\hline Alguma parte do tempo & $14(29,78)$ & 0 & \\
\hline Um pouco do tempo & $22(46,88)$ & $1(6,67)$ & \\
\hline Tempo nenhum & $2(4,25)$ & $14(93,33)$ & \\
\hline Total & $47(100)$ & $15(100)$ & \\
\hline Preocupado em tombar nas coisas & & & 0,090 \\
\hline O tempo todo & 0 & 0 & \\
\hline A maior parte do tempo & 0 & 0 & \\
\hline Boa parte do tempo & 0 & 0 & \\
\hline Alguma parte do tempo & $3(6,38)$ & 0 & \\
\hline Um pouco do tempo & $14(29,78)$ & $4(26,66)$ & \\
\hline Tempo nenhum & $30(63,82)$ & $11(73,34)$ & \\
\hline Total & $47(100)$ & $15(100)$ & \\
\hline Sente um peso para a família & & & 0,429 \\
\hline O tempo todo & 0 & 0 & \\
\hline A maior parte do tempo & 0 & 0 & \\
\hline Boa parte do tempo & $4(8,51)$ & 0 & \\
\hline Alguma parte do tempo & $18(38,29)$ & 0 & \\
\hline Um pouco do tempo & $3(6,38)$ & $2(13,33)$ & \\
\hline Tempo nenhum & $22(46,88)$ & $13(86,67)$ & \\
\hline Total & $47(100)$ & $15(100)$ & \\
\hline
\end{tabular}

Fonte: elaborada pelos próprios autores

*Nível de significância $(p<0,05)$ : Teste Exato de Fisher

No presente estudo, a maior parte das mulheres $(42,55 \%)$ sentia-se preocupada, em "um pouco do tempo", com a aparência (Tabela 5). Por outro lado, os homens apresentavam, assim, menos preocupações (26,66\%). Enquanto $46,88 \%$ das mulheres se preocupavam na escolha de roupas, apenas $6,67 \%$ dos homens tinham essa preocupação. Verificou-se, ainda, que enquanto $38,29 \%$ das mulheres se sentiam um peso para a família em "alguma parte do tempo", nenhum homem se sentia preocupado. No entanto, 2 participantes se sentiam um peso para a família, "em um pouco do tempo".

Esses resultados corroboram com os dados obtidos de um estudo clínico realizado nos Estados Unidos, onde se aplicou o VEINES QoL /Sym " nos participantes portadores de veias varicosas, e identificou-se que $75 \%$ dos 
participantes relataram preocupação com a aparência "um pouco do tempo" e $65 \%$ dos participantes relataram que a aparência influenciava na escolha das roupas ${ }^{(16)}$. Além disso, mais de $20 \%$ narraram se sentir um peso para a família ${ }^{(16)}$. Não foi observado, no entanto, distinção entre os sexos masculino e feminino(16).

Outro fator importante, que pode agravar a qualidade de vida dos pacientes com TVP, é a fadiga física e emocional ${ }^{(15)}$. Tais manifestações alteram a sua imagem corporal e interferem diretamente em suas relações sociais ${ }^{(15)}$. Alguns sentimentos como tristeza, ansiedade, raiva e vergonha são perceptíveis, considerando que esses sentimentos determinam um grande número de dificuldades, em relação ao autocuidado, sobretudo, quando percebem que são um "peso para a família" (Tabela 5).

De acordo com a Tabela 5, 29, 78\% das mulheres preocupavam "um pouco do tempo" em trombar nas coisas, após 40 dias de cirurgia. Pode-se inferir que, apesar de terem feito a cirurgia, ainda exibem veias varicosas que podem estar propícias a se transformarem em úlceras venosas abertas e, consequentemente, resultar numa insuficiência venosa crônica grave, isto é, incapacidade das válvulas venosas impulsionarem o sangue, em direção ascendente.

Importante destacar que, independente dos participantes, úlceras abertas ou não, algumas características físicas como o edema, por exemplo, ainda permaneceram em 22,6\% dos participantes pós-cirúrgicos. 0 edema pode indicar a presença de volume de líquidos excessivos nesses pacientes ${ }^{(11)}$. Em geral, é observado na região perimaleolar ou estende-se ao terço inferior da perna e está, frequentemente, associado à insuficiência venosa crônica. Devem ser empreendidas medidas para o controle do edema, já que prejudica o fluxo sanguíneo, a oxigenação e a nutrição dos tecidos.

Neste estudo, $57,15 \%$ dos participantes responderam que o edema "limita um pouco" as atividades do trabalho. Sendo assim, um acompanhamento pós- cirúrgico dos pacientes deve ser realizado, mesmo após um longo período de cirurgia, pois nem sempre acontece a melhora total da sua qualidade de vida, seja pelo número ou característica clínica de veias varicosas, que ainda permanecem, ou por fatores genéticos.

Os resultados deste estudo indicaram que, apesar da realização de cirurgia das veias varicosas, tais veias nem sempre foram totalmente eliminadas e, em casos graves, muitas vezes, não se conseguiu alcançar uma classificação clínica livre de riscos. Sinais e sintomas como inflamação, hiperpigmentação, edema e lipodermatoesclerose se apresentavam em alguns pacientes pós-cirúrgicos. Além disso, alguns participantes permaneceram, após a cirurgia, com limitações no trabalho e nas atividades de casa. Fatores psicológicos ainda permaneceram, no dia a dia, desses pacientes.

Por causa de todas as limitações físicas e psicossociais que os portadores de TVP apresentam, na vida diária, há uma necessidade, urgente, de adaptar os cuidados de saúde ao ambiente social dos indivíduos afetados e de impulsionar a busca por melhores condições de vida.

\section{CONCLUSÃO}

Os resultados do presente estudo contribuíram para uma avaliação sistemática da prática clínica institucional, e para intervenções pontuais implementadas na assistência, pois o tratamento cirúrgico não influenciava na diminuição da prevalência da IVC, pós cirúrgica e de suas características fisicas e clínicas, como a fisiopatologia e a presença de veias varicosas. Verificou-se, ainda, neste estudo, que a qualidade de vida, em sua maioria, não se restabeleceu após a cirurgia, perpetuando, somente de forma mais amena, sinais e sintomas da doença. Os resultados contribuíram, ainda, para a comunidade científica, uma vez que lacunas do conhecimento científico foram identificadas, como o impacto do tratamento cirúrgico na qualidade de vida dos pacientes com IVC. O questionário de qualidade de vida implantado possibilitou o conhecimento da situação de grupos populacionais específicos com amplo significado epidemiológico, dentro do contexto da assistência hospitalar. Estudos epidemiológicos futuros poderiam acompanhar pacientes com TVP, juntamente com toda a equipe multiprofissional, para que novas opções de tratamento e avaliação sejam consideradas, no pré e no pós cirúrgico.

\section{REFERÊNCIAS}

1- Barros BCS, Araújo A, Magalhães C, Barros R, Fiorelli $S$, Gatis R. Efficacy of varicose vein surgery with preservation of the great safenous vein. Rev Col Bras Cir. 2015;42(2):111-5.DOI: 10.1590/0100-699112015002008

2- Seidel AC, Coelho RL, Coelho M L, Belczar CEQ. 
Is vein damage the only cause of clinicals flower limb chronic venous insufficiency? J Vasc Bras. 2014;13(3): 162-7. DOI: 10.1590/jvb.2014.015

3- Sutzko DC, Obi AT, Kimball AS, Smith ME, Wakefield TW, Osborne NH. Clinical outcomes after varicose vein procedures in octogenarians within the Vascular Quality Initiative Varicose Vein Registry. J Vasc Surg Venous Lymphat Disord. 2018;6(4):464-70. DOI: 10.1016/j.jvsv.2018.02.008

4- Epstein D, Onida S, Bootun R, Ortega-Ortega $M$, Davies $A H$. Cost-effectiveness of current and emerging treatments of varicose veins. Value Health 2018;21(8):911-20. DOI: 10.1016/j.jval.2018.01.01

5- Torres SMSSO, Araújo RS, Costa IKF, Tibúrcio MP, Sousa AJG, Pergola-Marconato AM, et al. Health-related quality of life in patients with venous leg ulcer treated in primary care in Brazil and Portugal. PLoS One 2018; 13(4):e0195990. DOI: 10.1371/journal.pone.0195990

6- Lopes JF, Camacho ACLF, Silva RMCRA, Leite BS, Queiroz RS, Assis C RC. Impact of home visits on the functional capacity of patients with venous ulcers. Rev Bras Enferm. 2017;70(2):28793. DOI: 10.1590/0034-7167-2016-0291

7- Rossi F H, Volpato MG, Metzger PB, Beteli CB, Almeida BL, Rossi CBO, et al. Relationships between severity of signs and symptoms and quality of life in patients with chronic venous disease. J Vasc Bras. 2015;14(1): 22-8. DOI: 10.1590/1677-5449.20140039

8- Paty J, Elash CA, Turner-Bowker DM. Content validity for the VVSymQ $^{\circ}$ instrument: A new patient-reported outcome measure for the assessment of varicose veins symptoms. Patient 2017;10(1):51-63. DOI: 10.1007/s40271-0160183-y

9- Salmhofer W. Etiology, nomenclature and pathophysiology of chronic venous insufficiency. Wien Med Wochenschr. 2016;166(9/10):264-69. DOI: 10.1007/s10354-016-0466-x

10- Mahmoodi BK, Cushman M, Anne N I, Allison MA, Bos WJ, Brækkan SK, et al. Association of traditional cardiovascular risk factors with venous thromboembolism: An individual participant data meta-analysis of prospective studies. Circulation 2017;135(1):7-16. DOI: 10.1161 /CIRCULATIONAHA.116.024507

11- Nogueira G A, Oliveira BGRB, Santana RF, Cavalcanti ACD. Nursing diagnoses in patients with chronic venous ulcer: Observational study.Rev Eletr Enferm 2015;17(2):333-9. DOI:

\subsection{6/ree.v17i2.28782}

12- Okuhara A, Navarro TP, Procópio RJ, Leite JOM. Incidence of deep venous thrombosis and stratification of risk groups in a university hospital vascular surgery unit. J Vasc Bras. 2015;14(2):139-44. DOI: 10.1590/16775449.0071

13- Dias TYAF, Costa IKF, Melo MDM, Torres SMSGSO, Maia EMC. Quality of life assessment of patients with and without venous ulcer. Rev Latino-Am Enfermagem 2014;22(4):576-81. DOI: 10.1590/0104-1169.3304.2454

14- Nyquist $P$, Bautiste $C$, Jichici D, Burns J, Chhangani S, DeFilippis $M$, et al. Prophylaxis of venous thrombosis in neurocritical care patients: An evidence-based guideline: A statement for healthcare professionals from the neurocritical care society. Neurocrit Care 2016;24(1):47-60. DOI: $10.1007 /$ s12028-015-0221-y

15- Santos KFR, Silva PR, Ferreira VT, Domingues ARE, Simões IRA, Lima RS, et al. Quality of life of people with chronic ulcers. J Vasc Nurs. 2016;34(4): 131-6. DOI: 10.1016/j.jvn.2016.06.003

16- Mallick R, LAL BK, Daugherty C. Relationship between patient-reported symptoms, limitations in daily activities, and psychological impact in varicose veins.J Vasc Surg Venous Lymphat Disord. 2017;5(2):224-37. DOI: 10.1016/j.jvsv.2016.11.004

\begin{abstract}
Nota: Este estudo faz parte da monografia de final de curso intitulada "Perfil sociodemográfico de pacientes que necessitam de abordagem cirúrgica para varizes de membros inferiores" para obter o título de Bacharel em Enfermagem pela Universidade Federal dos Vales do Jequitinhonha e Mucuri. não é financiado por nenhuma agência de financiamento.
\end{abstract}

Recebido em: : 25/03/2019 Aprovado em: 13/12/2019

Endereço de correspondência:

Thabata Coaglio Lucas

Rodovia MGT36, Km 583,5000. Alto da Jacuba. CEP: 39100-000- Diamantina/MG - Brasil E-mail: thabataclucas@gmail.com 